\title{
Risk Factors for Carbapenemase-Producing Carbapenem-Resistant Enterobacteriaceae (CP-CRE) Acquisition Among Contacts of Newly Diagnosed CP-CRE Patients
}

\author{
Anat Schwartz-Neiderman, BSc; ${ }^{1}$ Tali Braun, BSc, MHA $;{ }^{1}$ Noga Fallach, MA; ${ }^{1}$ David Schwartz, PhD $;{ }^{2}$ \\ Yehuda Carmeli, MD, MPH; ${ }^{1,3}$ Vered Schechner, MD, MSc ${ }^{1}$
}

овјестіve. Carbapenemase-producing carbapenem-resistant Enterobacteriaceae (CP-CRE) are extremely drug-resistant pathogens. Screening of contacts of newly identified CP-CRE patients is an important step to limit further transmission. We aimed to determine the risk factors for CP-CRE acquisition among patients exposed to a CP-CRE index patient.

METHODs. A matched case-control study was performed in a tertiary care hospital in Israel. The study population was comprised of patients who underwent rectal screening for CP-CRE following close contact with a newly identified CP-CRE index patient. Cases were defined as positive tests for CP-CRE. For each case patient, 2 matched controls were randomly selected from the pool of contacts who tested negative for CP-CRE following exposure to the same index case. Bivariate and multivariate analyses were conducted using conditional logistic regression.

RESULTS. In total, 53 positive contacts were identified in 40 unique investigations (896 tests performed on 735 contacts) between October 6 , 2008, and June 7, 2012. bla $a_{\mathrm{KPC}}$ was the only carbapenemase identified. In multivariate analysis, risk factors for CP-CRE acquisition among contacts were (1) contact with an index patient for $\geq 3$ days (odds ratio [OR], 9.8; 95\% confidence interval [CI], 2.0-48.9), (2) mechanical ventilation (OR, 4.1; 95\% CI, 1.4-11.9), and (3) carriage or infection with another multidrug-resistant organism (MDRO; OR, 2.6; 95\% CI, 1.0-7.1). Among patients who received antibiotics, cephalosporins were associated with a lower risk of acquisition.

CONCLUSIONs. Patient characteristics (ventilation and carriage of another MDRO) as well as duration of contact are risk factors for CP-CRE acquisition among contacts. The role of cephalosporins requires further study.

Infect Control Hosp Epidemiol 2016;37:1219-1225

The emergence and spread of carbapenemase-producing carbapenem-resistant Enterobacteriaceae (CP-CRE) harboring the gene for Klebsiella pneumoniae carbapenemase $\left(b l_{\mathrm{KPC}}\right)$ is a major public health concern. Strains of $K$. pneumoniae and other Enterobacteriaceae harboring $b l a_{\mathrm{KPC}}$ are often extremely drug resistant and pose a significant therapeutic challenge. ${ }^{1,2}$ Infections by these strains have also been associated with poor clinical outcomes. ${ }^{3-5}$ Since they were originally reported in nosocomial outbreaks in the northeastern United States more than a decade ago, these strains have disseminated rapidly to become endemic pathogens in North America, and they are also widespread in Europe, Latin America, and Asia. ${ }^{6}$ Israel experienced a large outbreak beginning in 2006, and efforts to contain this outbreak are continuing. ${ }^{7,8}$

Data concerning the epidemiology of CP-CRE remain limited. Several case-control studies have identified healthcare-associated factors as predictors for isolation of CP-CRE among hospitalized patients. ${ }^{3,9,10}$ Molecular studies have demonstrated how clonal expansion and horizontal plasmid dissemination, either within the same species or, rarely, between species, underlie the spread of these multidrug-resistant pathogens. ${ }^{11-14}$

Strict contact isolation, physical separation of carriers from noncarriers, and use of dedicated staff are key components in containing CP-CRE in acute care hospitals. ${ }^{15}$ Active surveillance to promptly detect (and isolate) asymptomatic carriers is important measure to curtail transmission to other patients, as clinical cultures detect only a small portion of these carriers. ${ }^{16,17}$ In Israel, rectal screening is routinely performed for contacts of newly identified CP-CRE patients. This strategy has also been recommended in international guidelines. ${ }^{15}$ However, an explicit definition of who is a contact is lacking. Moreover, risk factors that explain why some contacts of a given index patient acquire CP-CRE and others do not have not yet been elucidated. In this study, we aimed to identify risk factors for acquisition of CP-CRE among patients exposed to an index patient colonized or infected with CP-CRE.

Affiliations: 1. Division of Epidemiology and Preventive Medicine, Tel Aviv Sourasky Medical Center, Tel Aviv, Israel; 2. Laboratory for Clinical Microbiology, Tel-Aviv Sourasky Medical Center, Tel Aviv, Israel; 3. National Center for Infection Control, Israel Ministry of Health, Tel Aviv, Israel.

Received February 25, 2016; accepted May 29, 2016; electronically published July 25, 2016

(c) 2016 by The Society for Healthcare Epidemiology of America. All rights reserved. 0899-823X/2016/3710-0013. DOI: 10.1017/ice.2016.153 


\section{METHODS}

\section{Study Setting, Patient Population, and Definitions}

Tel Aviv Sourasky Medical Center is a 1,400-bed, tertiary-care, teaching hospital in Tel Aviv, Israel. Cohorting with dedicated staff and strict contact isolation precautions have been enforced for all CP-CRE patients since mid-2007, and screening on admission of high-risk patients (eg, transfers from other hospitals or long-term care facilities) is routinely performed. We also screen contacts of newly identified CP-CRE patients, ie, contacts of patients who screened positive on admission but were not preemptively isolated, or contacts of patients who were identified as CP-CRE carriers by screening or clinical cultures during hospitalization. In our hospital, we use the following criteria for being a contact: (1) hospitalization in the same ward with a newly identified CP-CRE patient (prior to isolation) and (2) treatment by the same nursing staff. The population for our study was all adult inpatients who were screened for CP-CRE because of contact with a newly diagnosed CP-CRE index patient between October 2008 and June 2012. The study was approved by the hospital's institutional review board.

\section{Study Design}

A 2:1 matched case-control study was performed. We used a convenience sample; all patients who met the criteria for having a case of CP-CRE were included. The case group consisted of contacts who screened positive for CP-CRE during the investigation of a specific index patient. Patients with a positive CP-CRE screening test within 48 hours after admission were excluded from analysis because it was not possible to determine whether the CP-CRE was newly acquired or present on admission. The control group consisted of contacts who screened negative for CP-CRE during the investigation of a specific index patient. The basis for matching was participation in the same round of screening following exposure to the same index patient. This matching was done to control for characteristics of index patients that might affect transmissibility, such as bacterial load or diarrhea. ${ }^{18}$ Controls were randomly selected from the pool of negative contacts associated with each index patient.

\section{Microbiological Methods}

Rectal swabs were used for screening. Swabs were streaked onto selective MacConkey agar plates supplemented with $1 \mathrm{mg} / \mathrm{L}$ imipenem. Growing colonies were identified to the species level and tested for carbapenem resistance using the Vitek 2 system (bioMerieux, Marcy I'Etoile, France) and Etest for validation (ABBiodisk, Solna, Sweden). Enterobacteriaceae colonies growing on the selective plates were also tested by PCR for $b l a_{\mathrm{KPC}}$ throughout the study period and for $b l a_{\mathrm{NDM}}$ starting in October 2011 using methods previously described. ${ }^{19,20}$ Contacts who tested positive were only classified as cases if the carbapenemase detected by PCR matched that of the index patient, even if the organism differed. For example, if the contact and the index had carbapenemase-producing $K$. pneumoniae, but one had the $b l a_{\mathrm{KPC}}$ carbapenemase and the other had $b l a_{\mathrm{OXA}-48}$, the contact was not included as a case, ie, the contact was assumed not to have acquired the CP-CRE from that index. In contrast, if the contact had KPC-producing E. coli and the index had KPC-producing $K$. pneumoniae, then the contact was included as a having a case of CP-CRE, based on the assumption that he acquired KPC by horizontal gene transfer. ${ }^{12-14}$ In a few cases, $K$. pneumoniae isolates from the index patients were identified at another institution as carbapenemase producers by means of modified Hodge test without polymerase chain reaction testing (PCR). For this study, they were assumed to be $b l a_{\mathrm{KPC}}$ positive, because, during the study period, $b l a_{\mathrm{KPC}}$ was almost exclusively the mechanism of resistance among carbapenemase-producing K. pneumoniae in Israel. $^{21}$

\section{Data Collection}

Data were extracted from the contact patients' medical records and from the hospital's clinical and laboratory databases according to a pre-prepared questionnaire. The contact period was defined as the number of days that the index case and the contact shared the same ward. We created a dichotomous variable of $<3$ days or $\geq 3$ days; the cutoff point was chosen using the KolmogorovSmirnov goodness-of-fit test. Patient-related variables that were analyzed as possible predictors for CP-CRE acquisition included demographic characteristics (age and gender), specific comorbid conditions (diabetes mellitus, cardiovascular disease, chronic lung disease, renal disease, liver disease, neurologic disease, active malignancy), admission from a long-term care facility (LTCF) or another hospital, debilitated functional status (ie, fully dependent in activities of daily living ${ }^{22}$ ), ICU stay before screening, invasive devices (ie, central venous catheter, Foley catheter, mechanical ventilation), carriage or infection with another multidrug-resistant organism (ie, vancomycin-resistant Enterococci [VRE], methicillin-resistant Staphylococcus aureus [MRSA], ceftazidimeresistant Acinetobacter baumannii) or Clostridium difficile, and hospitalization in the previous 3 months. We also collected data related to receipt of any antibiotic or specific categories of antibiotics during hospitalization. Variables related to a contact's exposure to the index patient included the contact period and whether the index patient and the contact shared the same room.

\section{Statistical Analysis}

The association between presumptive predictors (not including exposure to individual antimicrobial agents) and having a case of CP-CRE was first examined by bivariate analysis using conditional logistic regression. The odds ratios (ORs) and their corresponding 95\% confidence intervals (CIs) were calculated. Results with $P \leq .05$ were considered statistically significant. Covariates with a $P \leq .10$ in bivariate analysis were considered for the multivariate model. A multivariate conditional logistic regression model was constructed using stepwise selection (with 
entry and stay criteria of $P \leq .15)$. We chose the model with the lowest Akaike information criterion (AIC) value. To assess the impact of exposure to specific antimicrobial classes, we re-ran the multivariate model, adding a different class each time. To create the final model, we repeated the stepwise selection including the antibiotic classes that were significant $(P \leq .1)$. All statistical analyses were conducted with SAS version 9.2 (SAS Institute, Cary, NC).

\section{RES U L T S}

In total, 319 investigations were conducted between October 2008 and June 2012 following the identification of new CPCRE index patients. In total, 3,158 were screened. In 40 investigations (896 tests performed on 735 contacts), at least 1 positive contact was identified by rectal screening (range: $1-4$ positive contacts per investigation). Overall, $53 \mathrm{CP}$-CRE screen-positive contacts and 453 screen-negative contacts were identified in these 40 investigations. The 53 positive contacts (cases) and 106 negative contacts ( 2 matched controls for each case) were included for analysis.

Table 1 summarizes the microbiological test results of the 40 index patients and their 53 corresponding positive contacts. $b l a_{\mathrm{KPC}}$ was the only carbapenemase identified in both groups. Table 2 outlines the characteristics of the 53 cases and their 106 matched controls. In bivariate matched analysis, patient characteristics that differed significantly between cases and controls included: mechanical ventilation during hospitalization $(42 \%$ vs $21 \%, P=.004)$, receipt of any antibiotic during hospitalization ( $83 \%$ vs $65 \%, P=.021)$, and being a carrier of another MDRO (40\% vs $15 \%, P<.001)$. Concerning the exposure to the index patient, the contact period differed between cases and controls, but sharing a room did not.

The initial multivariate analysis (not including specific antibiotics) identified 3 independent risk factors for CP-CRE acquisition among contacts: carriage or infection with another MDRO (OR, 2.3; 95\% CI, 0.9-6.0; $P=.081$ ), mechanical ventilation (OR, 2.3; 95\% CI, 0.9-6.0; $P=.072)$, and contact period of $\geq 3$ days (OR, 7.4; 95\% CI, 1.6-34.1; $P=.010$ ).
Our analysis of exposure to individual antibiotic classes, after adjustment for the 3 variables noted above, is presented in Table 3. CP-CRE acquisition tended to be associated with prior exposure to carbapenems; however, this did not reach statistical significance (OR, 4.0; 95\% CI, 0.8-19.0; $P=.085)$, while exposure to cephalosporins was protective (OR, $0.2 ; 95 \%$ CI, 0.1-0.6; $P=.005)$. Stepwise selection that included these 2 antibiotic classes and the 3 significant variables identified previously were used in our final model (Table 4). MDRO infection or carriage, mechanical ventilation, and contact period of $\geq 3$ days increased the risk of CP-CRE acquisition, and receipt of cephalosporins was protective.

To further explore the effect of cephalosporins we analyzed narrow-spectrum (first and second generation) and broadspectrum (third and fourth generation) cephalosporins separately. The effect was protective for both subclasses (data not shown). We also conducted a subgroup analysis to determine whether the effect of cephalosporin use was different if given alone or with other antibiotics. The effect was qualitatively different, although not statistically significant. When we compared patients who received only cephalosporins $(n=28)$ to patients who received no antibiotics $(n=44)$, cephalosporins increased the risk of CP-CRE acquisition (OR, 1.23; 95\% CI, 0.38-4.01). However, when we compared patients who received cephalosporins plus another antibiotic, either concurrently or at another time during this hospitalization $(n=19)$ to patients who received antibiotics that were not cephalosporins $(\mathrm{n}=68)$, cephalosporins were protective (OR, $0.49 ; 95 \%$ CI, 0.17-1.44).

We calculated how many positive contacts had at least 1 of the 3 risk factors identified by our model (not including cephalosporins). Of the 53 cases, all but 2 had at least 1 risk factor (ie, contact period of $\geq 3$ days, ventilation, or another MDRO). Among the 106 controls, 31 (29\%) had none of these risk factors.

\section{DISCUSSION}

Cohorting all CP-CRE carriers is a key component in controlling the spread of these MDROs in healthcare settings.

t A в LE 1. Microbiological Characteristics of CP-CRE Among the 40 Index Patients and Their 53 Corresponding Positive Contacts

\begin{tabular}{|c|c|c|c|c|c|}
\hline \multicolumn{3}{|c|}{ Index Patients $(\mathrm{n}=40)$} & \multicolumn{3}{|c|}{ Positive Contacts $(\mathrm{n}=53)$} \\
\hline No. of Isolates & Bacterium & Carbapenemase & No. of Isolates & Bacterium & Carbapenemase \\
\hline 27 & K. pneumoniae & $b l a_{\mathrm{KPC}}$ & 39 & K. pneumoniae & $b l a_{\mathrm{KPC}}$ \\
\hline 3 & K. pneumoniae & N/A & 3 & K. pneumoniae & $b l a_{\mathrm{KPC}}$ \\
\hline 4 & $N A$ & $b l a_{\mathrm{KPC}}$ & 4 & K. pneumoniae & $b l a_{\mathrm{KPC}}$ \\
\hline 4 & K. pneumoniae & $b l a_{\mathrm{KPC}}$ & 4 & E. coli/ Enterobacter spp. & $b l a_{\mathrm{KPC}}$ \\
\hline 1 & K. pneumoniae & NA & 1 & E. coli & $b l a_{\mathrm{KPC}}$ \\
\hline \multirow[t]{2}{*}{$1^{\mathrm{a}}$} & K. pneumoniae & $b l a_{\mathrm{KPC}}$ & 1 & E. coli & $b l a_{\mathrm{KPC}}$ \\
\hline & & & 1 & K. pneumoniae & $b l a_{\mathrm{KPC}}$ \\
\hline
\end{tabular}

${ }^{a}$ This index patient had two positive contacts, each with a different organism.

NOTE. CP-CRE, carbapenemase-producing carbapenem-resistant Enterobacteriaceae; NA, not available. 
TABLE 2. Comparison of Cases and Controls (Univariate and Bivariate Analyses)

\begin{tabular}{|c|c|c|c|c|}
\hline \multirow[b]{2}{*}{ Covariate } & \multicolumn{2}{|c|}{ No. of Patients (\%) } & \multirow[b]{2}{*}{ OR $(95 \% \mathrm{CI})$} & \multirow[b]{2}{*}{$P$ Value } \\
\hline & $\begin{array}{l}\text { Positive Contacts } \\
\quad(\mathrm{N}=53),(\%)\end{array}$ & $\begin{array}{l}\text { Negative Contacts } \\
(\mathrm{N}=106),(\%)\end{array}$ & & \\
\hline \multicolumn{5}{|l|}{ Patient characteristics } \\
\hline Age, mean $\pm S D, y$ & $74.0 \pm 17.1$ & $71.6 \pm 18.5$ & & 0.449 \\
\hline Female sex & $26(49.1)$ & $55(51.9)$ & $1.1(0.6-2.2)$ & 0.731 \\
\hline Diabetes mellitus & $22(41.5)$ & $39(36.8)$ & $1.2(0.6-2.4)$ & 0.567 \\
\hline Cardiovascular disease & $41(77.4)$ & $68(64.2)$ & $2.0(0.9-4.3)$ & 0.094 \\
\hline Chronic lung disease & $14(26.4)$ & $14(13.2)$ & $2.2(1.0-4.9)$ & 0.053 \\
\hline Renal disease & $17(32.1)$ & $27(25.5)$ & $1.4(0.7-2.9)$ & 0.376 \\
\hline Liver disease & $2(3.8)$ & $9(8.5)$ & $0.4(0.1-1.9)$ & 0.226 \\
\hline Neurologic disease & $16(30.2)$ & $30(28.3)$ & $1.1(0.5-2.2)$ & 0.814 \\
\hline Active malignancy & $5(9.4)$ & $20(18.9)$ & $0.4(0.1-1.2)$ & 0.113 \\
\hline Arrived from LTCF or hospital & $15(28.3)$ & $19(17.9)$ & $1.7(0.8-3.5)$ & 0.160 \\
\hline Debilitated functional status & $15(28.3)$ & $25(23.6)$ & $1.3(0.6-2.6)$ & 0.533 \\
\hline Norton score $\geq 10$ & $37(69.8)$ & $73(68.9)$ & $1.0(0.5-2.1)$ & 0.905 \\
\hline ICU stay ${ }^{\mathrm{a}}$ & $14(26.4)$ & $21(19.8)$ & $1.7(0.7-4.5)$ & 0.260 \\
\hline Mechanical ventilation $^{\mathrm{a}}$ & $22(41.5)$ & $22(20.8)$ & $3.3(1.4-7.4)$ & 0.004 \\
\hline Surgery ${ }^{*}$ & $13(24.5)$ & $24(22.6)$ & $1.1(0.5-2.7)$ & 0.768 \\
\hline Urinary Foley catheter $^{\mathrm{a}}$ & $26(49.1)$ & $45(42.5)$ & $1.3(0.7-2.5)$ & 0.440 \\
\hline $\mathrm{CVC}^{\mathrm{a}}$ & $17(32.1)$ & $27(25.5)$ & $1.9(0.7-5.6)$ & 0.222 \\
\hline Carriage or infection with another MDRO & $21(39.6)$ & $16(15.1 \%)$ & $4.0(1.7-9.2)$ & 0.001 \\
\hline Hospitalization in the previous 3 mo & $29(54.7)$ & $46(43.4 \%)$ & $1.6(0.8-3.2)$ & 0.171 \\
\hline Receipt of any antibiotic ${ }^{\mathrm{a}}$ & $44(83.0)$ & $69(65.1 \%)$ & $2.6(1.2-5.8)$ & 0.021 \\
\hline \multicolumn{5}{|l|}{ Contact with the index patient } \\
\hline Contact period $\geq 3 \mathrm{~d}$ & $44(83.0)$ & $63(59.4 \%)$ & $10.3(2.3-45.1)$ & 0.002 \\
\hline Shared room & $23(43.4)$ & $34(32.1 \%)$ & $2.1(0.9-5.0)$ & 0.089 \\
\hline
\end{tabular}

${ }^{\mathrm{a}}$ During current hospitalization.

NOTE. SD, standard deviation; LTCF, long-term care facility; ICU, intensive care unit; CVC, central venous catheter; MDRO, multidrug-resistant organism (ie, VRE, MRSA, Ceftazidime-resistant Acinetobacter baumannii, or Clostridium difficile).

Table 3. Adjusted Antibiotic Risk Factors for CP-CRE Acquisition Among Contacts

\begin{tabular}{lcccc}
\hline & \multicolumn{2}{c}{ No. of Patients (\%) } & & \\
\cline { 2 - 4 } Covariate & $\begin{array}{c}\text { Positive } \\
\mathrm{N}=53),(\%)\end{array}$ & $\begin{array}{c}\text { Negative } \\
(\mathrm{N}=106),(\%)\end{array}$ & OR (95\% CI) & $P$ Value \\
\hline Penicillins & $6(11.3)$ & $12(11.3)$ & $0.5(0.1-2.1)$ & .328 \\
Cephalosporins & $12(22.6)$ & $35(33.0)$ & $0.2(0.1-0.6)$ & .005 \\
$\beta$-lactam/ $\beta$-lactamase inhibitor combinations & $2(3.8)$ & $4(3.8)$ & $1.6(0.2-12.3)$ & .668 \\
Carbapenems & $6(11.3)$ & $5(4.7)$ & $4.0(0.8-19.9)$ & .085 \\
Aminoglycosides & $4(7.5)$ & $6(5.7)$ & $1.6(0.3-8.0)$ & .578 \\
Fluoroquinolones & $8(15.1)$ & $13(12.3)$ & $0.9(0.3-2.7)$ & .839 \\
Macrolides & $1(1.9)$ & $1(0.9)$ & $0.4(0.0-13.1)$ & .591 \\
Glycopeptides & $8(15.1)$ & $6(5.7)$ & $1.8(0.4-7.6)$ & .409 \\
Other antibiotics & $22(41.5)$ & $24(22.6)$ & $1.3(0.6-3.0)$ & .474 \\
\hline
\end{tabular}

NOTE. CP-CRE, carbapenemase-producing carbapenem-resistant Enterobacteriaceae.

Relying solely on clinical cultures detects only a fraction of the actual colonized population. Therefore, active surveillance of high-risk patients, including patients in contact with an index case, is strongly recommended. ${ }^{15}$
We identified 3 risk factors for CP-CRE acquisition among contacts: (1) mechanical ventilation, (2) colonization or infection with another MDRO, and (3) contact with the index patient for $\geq 3$ days. Several studies have cited mechanical 
TABLE 4. Risk Factors for CP-CRE Acquisition Among Contacts: A Multivariate Model Including Exposure to Specific Antibiotic Classes

\begin{tabular}{lll}
\hline Covariate & OR (95\% CI) & $P$ Value \\
\hline Contact period $\geq 3$ days & $9.8(2.0-48.9)$ & .005 \\
Receipt of cephalosporins & $0.2(0.1-0.6)$ & .005 \\
Mechanical ventilation & $4.1(1.4-11.9)$ & .011 \\
Carriage or infection with another MDRO & $2.6(1.0-7.1)$ & .062 \\
\hline
\end{tabular}

NOTE. CP-CRE, carbapenemase-producing carbapenem-resistant Enterobacteriaceae; OR, odds ratio; MDRO, multidrug-resistant organism (ie, VRE, MRSA, ceftazidime-resistant Acinetobacter baumannii, or Clostridium difficile).

ventilation as a risk factor for CRE acquisition. ${ }^{23,24}$ The pathway by which ventilation increases the risk of CP-CRE acquisition is uncertain. It may be that ventilation is a marker for severe illness; severely ill patients may require more frequent contact with staff, who may act as a vector of transmission. On the other hand, other variables that suggest a need for frequent staff contact, such as ICU stay and debilitated functional status, were not associated with CP-CRE acquisition in our study.

The fact that carriage or infection with another MDRO is a risk factor for acquiring CP-CRE implies that some patients are highly prone to acquire MDROs of all types. Notably, even though these patients would already have been on contact precautions because of their other MDROs, they nevertheless acquired CP-CRE. This may simply represent a lapse in infection control measures or it may suggest that these "MDRO prone patients" require additional preventive measures, such as interventions to restore their microbiome. ${ }^{25}$ Future research should better characterize this group and identify the measures needed to protect them.

Other case-control studies that have analyzed risk factors for CP-CRE acquisition focused on characteristics of susceptible individuals. $^{23,26,27}$ However, acquisition events may be influenced by the characteristics of the carrier who is the transmitter. We showed in a previous study that $20 \%$ of the carriers are responsible for $80 \%$ of the environmental spread. ${ }^{18}$ Moreover, specific time and ward characteristics may influence the risk of acquisition, eg, compliance with hand hygiene and other infection control measures, efficacy of environmental cleaning, and staff-to-patient ratio. The strength of this study is that we compared the risk of acquisition between patients that were exposed to the same carrier under the same circumstances. We found that a contact period of at least 3 days was a risk factor for CP-CRE acquisition, while sharing a room was not. Surprisingly, sharing a room was not significant; the CDC specifically identifies roommates as contacts who should be screened. ${ }^{15}$ The risk might stem not simply from being in the same room but from the number of transmission opportunities between the index and the contact, generally via vectors (the contaminated hands of staff) or shared equipment. Measurement of transmission opportunities is complicated and requires detailed observation of staff and patient activity.
Exposure to antimicrobials has been identified in many studies as an important risk factor for CP-CRE colonization and infection; however, great variability exists between studies when individual antimicrobial agents are considered..$^{3,4,9,10,26-28}$ These discrepancies might be related at least in part to different definitions of antimicrobial exposure and the possibility of uncontrolled confounders in retrospective studies. ${ }^{29}$ In our study, the small number of patients who received each specific class of antibiotic limited our ability to find statistically significant associations. Exposure to carbapenems was marginally associated with an increased risk of CP-CRE acquisition; however, in a multivariate model, this exposure was not identified as an independent risk factor. Our finding agrees with the results of other studies of acquisition of resistant Enterobacteriaceae. ${ }^{10,26}$ Exposure to cephalosporins was unexpectedly identified as a protective factor $(\mathrm{OR}, 0.2)$. However, in a subgroup analysis that isolated the effect of cephalosporins by comparing patients who received only cephalosporins to those who received no antibiotics, cephalosporins emerged as a risk factor (although not statistically significant). More precise measurement of antibiotic use that includes dose, duration, and drug combinations may help clarify the association between antibiotic exposure and CP-CRE acquisition.

We are aware of a number of limitations to our study. First, we assumed that all cases acquired CP-CRE because of their contact with the index patient. Alternatively, patients may have already been carriers on admission or may have acquired CP-CRE from someone other than the index patient. The first possibility is unlikely because we screened high-risk patients (ie, those admitted from another hospital or LTCF and those hospitalized in the previous year) on admission. The probability of a low-risk patient being positive on admission is extremely low. In a study conducted in an ICU in Greece, no patients admitted to the ICU directly from the ER without previous hospitalization screened positive for KPC-producing K. pneumoniae, while $31 \%$ of patients hospitalized in the last year were positive on admission. ${ }^{30}$ The possibility that a case acquired CP-CRE not from the index patient but from another known CP-CRE carrier is also improbable, because of strict compliance with cohorting and dedicated staffing for all known CP-CRE carriers in our hospital. Finally, given our extensive screening protocols, it is unlikely that an unknown carrier could be the source of transmission. A second limitation of our study is that the only carbapenemase 
identified was $b l a_{\mathrm{KPC}}$; therefore, our findings may not be generalizable to settings where other carbapenemases are predominant. A third limitation is that although we have identified predictors of CP-CRE acquisition, they may not be readily available. For example, it might be difficult to determine the contact period between an index case and contacts. However, many institutions are now using electronic medical records that make such epidemiological investigations easier.

Our findings have implications for clinical practice. First, we might limit screening to contacts with any of the risk factors we have identified. Indeed, we screened more than 3,000 contacts to detect 53 acquisitions, a yield of $<2 \%$. We calculated that selective screening based on risk factors would have decreased the number of contacts screened by $30 \%$, but $4 \%$ of positive contacts would have been missed. However, if maximum sensitivity is considered crucial to prevent further transmission, an alternate approach would be to screen all contacts and preemptively isolate those with risk factors. Second, our study identified predictors of CP-CRE acquisition among contacts. Potentially, such predictors can be used to direct clinical care, for example, the choice of empiric antibiotic therapy for exposed patients with signs of infection while screening results are pending. Repeat studies confirming our findings are needed before such recommendations can be made as a means of promoting antibiotic stewardship.

\section{ACKNOWLEDGMENTS}

The authors would like to thank Dr. Elizabeth Temkin for her helpful comments on this work.

Financial support: No financial support was provided relevant to this article.

Potential conflicts of interest: Y.C. reports that during the last 3 years, he personally, his laboratory, and studies that he has conducted received grants, honoraria, travel support, consulting fees, and other forms of financial support from the following companies: Allecra Therapeutics, AstraZeneca, Biomerieux SA, DaVolterra, Durata Therapeutic, Valneva SE, Merck, PPD, Rempex Pharmaceuticals, Syntezza Bioscience, and Takeda Pharmaceutical. A.S.N., T.B., N.F., D.S., and V.S. report no conflicts of interest relevant to this article.

Address correspondence to Vered Schechner, MD, MSc, Division of Epidemiology and Preventive Medicine, Tel Aviv Sourasky Medical Center, 6 Weizmann St., Tel Aviv 64239, Israel (vereds@tlvmc.gov.il).

\section{REFERENCES}

1. Falagas ME, Lourida P, Poulikakos P, Rafailidis PI, Tansarlia GS. Antibiotic treatment of infections due to carbapenem-resistant Enterobacteriaceae: systematic evaluation of the available evidence. Antimicrob Agents Chemother 2014;58:654-663.

2. Tumbarello M, Trecarichi EM, De Rosa FG, et al. Infections caused by KPC-producing Klebsiella pneumoniae: differences in therapy and mortality in a multicentre study. J Antimicrob Chemother 2015;70:2133-2143.

3. Schwaber MJ, Klarfeld-Lidji S, Navon-Venezia S, Schwartz D, Leavitt A, Carmeli Y. Predictors of carbapenem-resistant Klebsiella pneumoniae acquisition among hospitalized adults and effect of acquisition on mortality. Antimicrob Agents Chemother 2008;52:1028-1033.
4. Gasink LB, Edelstein PH, Lautenbach E, Synnestvedt M, Fishman NO. Risk factors and clinical impact of Klebsiella pneumoniae carbapenemase-producing K. pneumoniae. Infect Control Hosp Epidemiol 2009;30:1180-1185.

5. Fraenkel-Wandel Y, Raveh-Brawer D, Wiener-Well Y, Yinnon AM., Assous MV. Mortality due to blaKPC Klebsiella pneumoniae bacteraemia. J Antimicrob Chemother 2016;71:1083-1087.

6. Pitout JD, Nordmann P, Poirel L. Carbapenemase-producing Klebsiella pneumoniae, a key pathogen set for global nosocomial dominance. Antimicrob Agents Chemother 2015;59:5873-5884.

7. Schwaber MJ, Lev B, Israeli A, et al. Containment of a country-wide outbreak of carbapenem-resistant Klebsiella pneumoniae in Israeli hospitals via a nationally implemented intervention. Clin Infect Dis 2011;52:848-855.

8. Schwaber MJ, Carmeli Y. An ongoing national intervention to contain the spread of carbapenem-resistant enterobacteriaceae. Clin Infect Dis 2014;58:697-703.

9. Wiener-Well Y, Rudensky B, Yinnon AM, et al. Carriage rate of carbapenem-resistant Klebsiella pneumoniae in hospitalised patients during a national outbreak. J Hosp Infect 2010;74:344-349.

10. Patel G, Huprikar S, Factor SH, Jenkins SG, Calfee DP. Outcomes of carbapenem-resistant Klebsiella pneumoniae infection and the impact of antimicrobial and adjunctive therapies. Infect Control Hosp Epidemiol 2008;29:1099-1106.

11. Kitchel B, Rasheed JK, Patel JB, et al. Molecular epidemiology of KPC-producing Klebsiella pneumoniae isolates in the United States: clonal expansion of multilocus sequence type 258. Antimicrob Agents Chemother 2009;53:3365-3370.

12. Goren MG, Carmeli Y, Schwaber MJ, Chmelnitsky I, Schechner V, Navon-Venezia S. Transfer of carbapenem-resistant plasmid from Klebsiella pneumoniae ST258 to Escherichia coli in patients. Emerg Infect Dis 2010;16:1014-1017.

13. Adler A, Khabra E, Paikin S, Carmeli Y. Dissemination of the blaKPC gene by clonal spread and horizontal gene transfer: comparative study of incidence and molecular mechanisms. 2016. [Epub ahead of print].

14. Sidjabat HE, Silveira FP, Potoski BA, et al. Interspecies spread of Klebsiella pneumoniae carbapenemase gene in a single patient. Clin Infect Dis 2009;49:1736-1738.

15. Facility guidance for control of carbapenem-resistant Enterobacteriaceae (CRE): November 2015 update-CRE Toolkit. Centers for Disease Control and Prevention website. http://www.cdc.gov/ hai/pdfs/cre/CRE-guidance-508.pdf. Accessed on January 8, 2016.

16. Ben-David D, Maor Y, Keller N, et al. Potential role of active surveillance in the control of a hospital-wide outbreak of carbapenem-resistant Klebsiella pneumoniae infection. Infect Control Hosp Epidemiol 2010;31:620-626.

17. Calfee D, Jenkins SG. Use of active surveillance cultures to detect asymptomatic colonization with carbapenem-resistant Klebsiella pneumoniae in intensive care unit patients. Infect Control Hosp Epidemiol 2008;29:966-968.

18. Lerner A, Adler A, Abu-Hanna J, Cohen Percia S, Kazma Matalon M, Carmeli Y. Spread of KPC-producing carbapenem-resistant Enterobacteriaceae: the importance of super-spreaders and rectal KPC concentration. Clin Microbiol Infect 2015;21(470):e471-e477.

19. Schechner V, Straus-Robinson K, Schwartz D, et al. Evaluation of PCR-based testing for surveillance of KPC-producing carbapenem-resistant members of the Enterobacteriaceae family. J Clin Microbiol 2009;47:3261-3265. 
20. Ellington MJ, Kistler J, Livermore DM, Woodford N. Multiplex PCR for rapid detection of genes encoding acquired metallobetallactamases. J Antimicrob Chemother 2007;59:321-322.

21. Navon-Venezia S, Leavitt A, Schwaber MJ, et al. First report on a hyperepidemic clone of KPC-3-producing Klebsiella pneumoniae in Israel genetically related to a strain causing outbreaks in the United States. Antimicrob Agents Chemother 2009;53:818-820.

22. Katz S, Ford AB, Moskowitz RW, Jackson BA, Jaffe MW. Studies of illness in the aged. The index of ADL: a standardized measure of biological and psychosocial function. JAMA 1963;185:914-919.

23. Swaminathan M, Sharma S, Poliansky Blash S, et al. Prevalence and risk factors for acquisition of carbapenem-resistant Enterobacteriaceae in the setting of endemicity. Infect Control Hosp Epidemiol 2013;34:809-817.

24. Chitnis AS, Caruthers PS, Rao AK, et al. Outbreak of carbapenem-resistant enterobacteriaceae at a long-term acute care hospital: sustained reductions in transmission through active surveillance and targeted interventions. Infect Control Hosp Epidemiol 2012;33:984-992.
25. Tosh PK, McDonald LC. Infection control in the multidrugresistant era: tending the human microbiome. Clin Infect Dis 2012;54:707-713.

26. Ahn JY, Song JE, Kim MH, et al. Risk factors for the acquisition of carbapenem-resistant Escherichia coli at a tertiary care center in South Korea: a matched case-control study. Am J Infect Control 2014;42:621-625.

27. Ling ML, Tee YM, Tan SG, et al. Risk factors for acquisition of carbapenem resistant Enterobacteriaceae in an acute tertiary care hospital in Singapore. Antimicrob Resist Infect Control 2015;4:26.

28. Kwak YG, Choi SH, Choo EJ, et al. Risk factors for the acquisition of carbapenem-resistant Klebsiella pneumoniae among hospitalized patients. Microb Drug Resist 2005;11:165-169.

29. Schechner V, Temkin E, Harbarth S, Carmeli Y, Schwaber MJ. Epidemiological interpretation of studies examining the effect of antibiotic usage on resistance. Clin Microbiol Rev 2013;26:289-307.

30. Papadimitriou-Olingeris M, Marangos M, Fligou F, et al. Risk factors for KPC-producing Klebsiella pneumoniae enteric colonization upon ICU admission. J Antimicrob Chemother 2012;67:2976-2981. 